\title{
The Performance of Saturated Packet Queue System On Broadband PLC Network
}

\author{
${ }^{1}$ Basuki Rahmat, ${ }^{2}$ Rinaldy Dalimi, ${ }^{3}$ Kalamullah Ramli \\ Department of Electrical Engineering Faculty of Engineering, University of Indonesia, Depok \\ Indonesia 16424
}

\begin{abstract}
The latest development of Broadband Power Line Communication Network may offer a platform for synchrophasor-sensor network in power distribution networks to establish a smart grid. Random data traffic passing through the slave-station of synchrophasor-sensor network can be viewed as packet entering of a queue. Since the length of queue is limited the packet loss may occur. This negatively affects the queuing system performance. Our numerical evaluation shows that for 1,000 random packet arrival, with 14-150 Mbps arrival rate, 100 Mbps service rate, and 64 Mbit buffer capacities, there is average packet loss of $6.75 \%$. If the buffer capacity is increased to $128 \mathrm{Mbit}$, then average of packet loss is reduced to $6.69 \%$. Our numerical test shows that the option to increase queue service rate may reduce packet loss probability more significantly than to increase the buffer capacity of the queue system.
\end{abstract}

Keywords - Broadband PLC; Network; Packet loss; Queue

\section{Introduction}

Telecommunication network over the power system is one of the important support facilities in power systems operation, especially for monitoring and controlling functions over wide-area coverage of power distribution network. Such networks can be viewed as neural network of power systems [1, 6]. Latest Broadband Power Line Communication (Broadband PLC) technology with HomePlug AV or IEEE P1901 standard could be regarded as one of the broadband communications infrastructures to deploy of synchrophasor-sensor network to establish a smart distribution grid [11]. Diagram model of such monitor and control system of power distribution over the Broadband PLC network is shown in Figure 1.

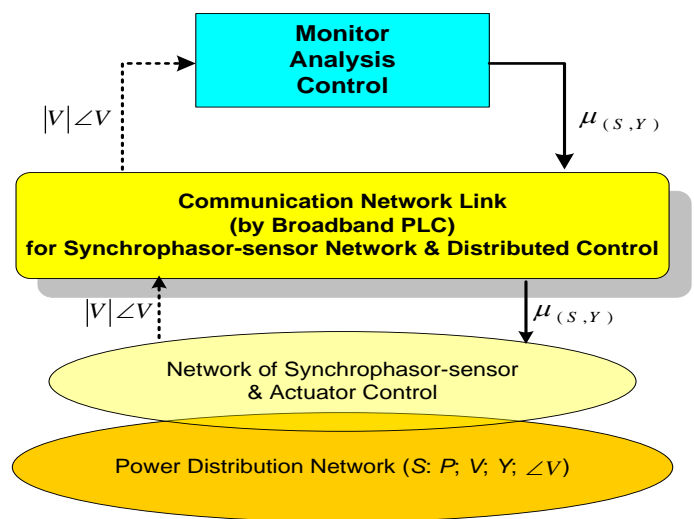

Fig.1. Principle of Monitor and Control of Electric Power Distribution Network System

Assuming the data information from a number of synchcrophasor-sensors in the power distribution network are always provided and transmitted to the master station through slave-station, there is random queue of data packet (packet) traffic on slave-station. If there is disturbance on any part of power distribution network, it will have wide-ranging effect and trigger a number synchrophasor-sensor to generate and transmit the data simultaneously. As a result, the rate of packet arriving in a queue may exceed the service rate of the slavestation. Furthermore, due to the limited buffer capacity of a queue, the packet queue system will soon get saturated. This causes some loss of the new packet arrival, and thus the synchrophasor data integrity is no longer warranted [4].

On the other hand, the communication networks used as transports of monitor and control information of power systems have to comply with high data integrity and minimum packet loss requirement $[3,4]$. Hence, saturated queue effect becomes the focus in analyzing queuing system performance on the Broadband PLC network. 
In this research, we perform an analysis of saturated packet queuing system, based on a model of packet queuing system, and approached by Markov chain. Next, we conduct the parametric studies of queuing systems to acquire most suitable parameters to improve performance of packet queuing systems. The results of the analysis could be used as a basic consideration to maintain the performance of packet queue system on Broadband PLC network.

\section{Synchrophasor-Sensor Network Based on Broadband PLC Technology}

Figure 2 shows the diagram model of synchrophasor-sensor network based on Broadband PLC network within a hierarchical structure. This model fulfills the communication network requirement for monitoring and controlling power distribution network.

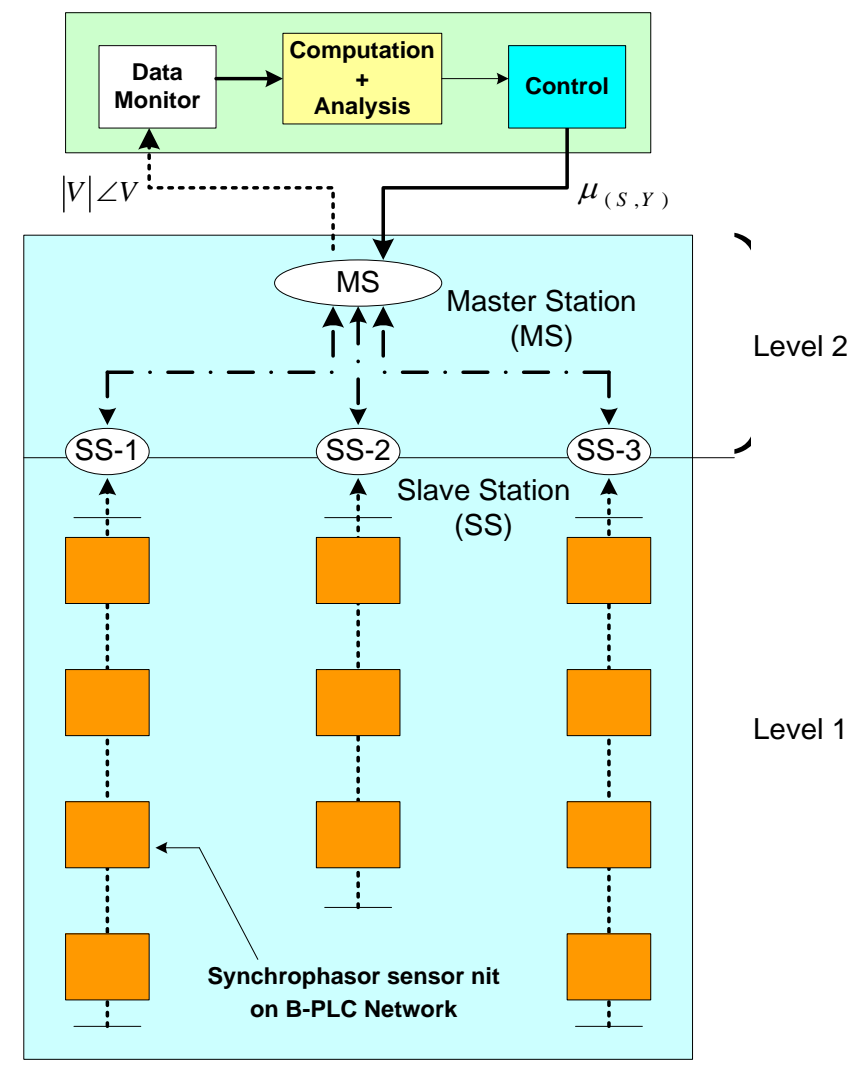

Fig.2. Structure of Synchrophasor-Sensor Network on Broadband-PLC Network

The first-level hierarchy is synchrophasor-sensor networks, which consist of a number synchrophasor-sensor and are divided into three network segments. In each of the segments, there are slave-station, namely SS-1, SS-2 and SS-3 that serve as gateway and coordinate the entire synchrophasor-sensor nodes on each network segment. The second level hierarchy is a central network, which consists of a master station (MS) and three slave-stations including SS-1, SS-2 and SS-3. Master station is a gateway and coordinates those networks. Each of synchrophasor-sensor nodes and station is purportedly based on Broadband PLC technology by a HomePlug AV(HPAV) standard, except for the master station. Master station is used as a unified communications technology to adapt with various of communication technology. Broadband PLC technology employed is HPAV standard with physical layer specification in Fast Fourier transforms - Orthogonal Frequency Division Multiplexing (FFT-OFDM) scheme and maximum of data bit rate up to $150 \mathrm{Mbps}[11,15]$.

Slave station is assumed to have multi inputs and limited buffer capacity with single output transmission channel into the master station, as shown in Figure 3. As a network gateway, slave station performs data collection from some synchrophasors-sensor to be transmitted into the master station. Hence, there is a queue of packet traffic on the slave station. Arrival of packet in a queue is randomly, so that is viewed as a stochastic process. Furthermore, packets departure is served by single output channel. For the data transmission over the output channel, the system employs Binary Phase Shift Keying (BPSK) and 1024_Quadrature Amplitude Modulation (QAM) of a modulation system. Both modulation systems are unified in an Orthogonal Frequency Division Multiple Access (OFDMA) of a scheme [15]. 


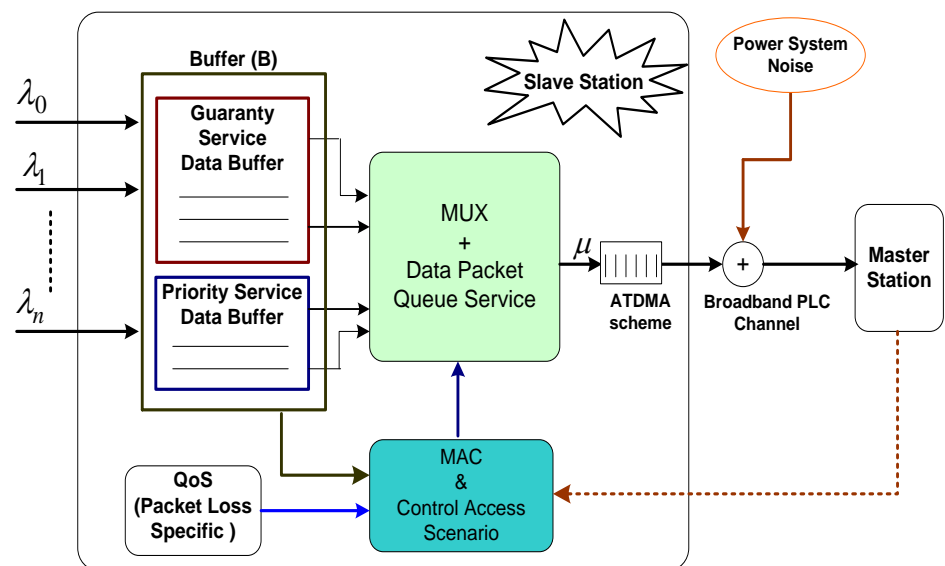

Fig. 3. Slave-station Diagram of Broadband PLC Network

\section{Traffic Control of Queue System}

\subsection{Coordination of Network HomePlug AV}

Simultaneously, packets transmission from a number stations or synchrophasor-sensor units may cause packet collision at shared transmission channel. Hence, network coordination is used on slave station to avoid the collision. Slave station acts as Connection Manager (CM) and serves as central coordinator (CCo) of the network. Slave station periodically broadcasts a beacon signal to the entire of network nodes for coordination purpose. Duration of a beacon period is covered on two periods of the cycle AC power which is equivalent to 0.04 seconds. At the same time, to synchronize of all network nodes, beacon signal is broadcasted throughout the network nodes at exactly the same time as "zero-intersection" on the AC power line. A beacon period consists of three parts, namely beacon slot, Contention phase slot, and phase Contention Free slot [15].

\subsection{Multiple Access Control}

Broadband PLC technology with HomePug AV standard is designed to apply a hybrid protocol which includes Carrier Sense Multiple Access/Collision Avoidance (CSMA/CA) and Time Division Multiple Access (TDMA) schemes. Both protocols are arranged in a frame of the beacon period to meet of access control requirement. The CSMA/CA scheme to handle of transmission service based on priority of services, whereas, the TDMA scheme to tackle of transmission service based on a guarantee of service such as bandwidth and delay and jitter the requirements $[5,15]$. CSMA/CA scheme set on Contention phase, whereas TDMA scheme established in Contention Free Phase.

Usage of the shared transmission channel in TDMA scheme may be ineffective, because of the random nature of transmission of packet from a number of sources. There is also possibility that large amount of data come from some sources simultaneously. Therefore, for the efficiency of shared transmission channel from the outputs of slave station to the master station in a TDMA scheme, an Asynchronous Time Division Multiple Access (ATDMA) technique is applied. In ATDMA, each of a transmission time slot is dedicated to data packets from a number of sources. There, each of packets arrivals is stored in a queue buffer waiting to obtain the access time slot to transmit to the master station. Furthermore, a number of packets in a queue buffer are simultaneously transmitted on one-time slot, and each of packet accompanied by additional header block, which contains both source and destination address bits and control bits of the transmission process.

However, if the buffer is full due to constant arrival of packets, especially when the arrival rate exceeds the service rate, packet loss may occur. Therefore, the saturated analysis of queuing system in the packet loss probability is an important issue in this work.

\section{Queuing of Packet Traffic on Broadband PLC Network}

As it is assumed on section II, arrival of packet in a queue is random. Therefore, the random variation of packet population in a queue buffer within slave station depends on the nature of arrival and departure of a queue. To determine the queuing system model of packet traffic, first a number of variables and parameters are defined, including $\lambda_{j} ; a_{m}^{j} ; n ; r_{i j} ; \mu_{j}$ and $t$, which state the quantities of arrival rate, batch of arrival, total packet population in the buffer, phase transition's rate, departure rate, and free time variables; where $i, j$ and $k$ express the phase of queuing system process. Further $m=0,1,2 \ldots, B$ express the size of packet arrival; $Q_{i j}(n, t)$ states of packet population on phase " $j "$ after the transition, from " $i$ " phase to " $j "$ phase. 


\subsection{Queuing System Model of Packet Traffic}

In accordance with the assumption of queuing system state as in section II, the queue of packet traffic may be expressed in the Kendal's form, as BMAP/M/1/B. This form shows the state of queuing system with the arrival as Batch Arrival Markov Process (BMAP) which is Multiphase Batch Poisson and single of queue server is exponentially, and limited buffer capacity. Thereby, packet population of a queue system at random continuously changes from a state to the next. Such randomly dynamics is approached by Markov chain as the model of the state of queue system according to the Kendal's form.

Furthermore, with $i$ and $j$ which are the adjacent phase of queue system state, we review on " $j$ " phase of queuing system state after the transition from " $i$ " phase, in transition rate at " $r_{i j}$ ". The assumption, on $t$ duration, are the packet arrival, denoted by $A_{t}=a_{m}^{j} \lambda_{j} t$ with $A=a_{m}^{j} \lambda_{j}$ as the arrival rate. The packets departure that is expressed by $C_{t}=\mu_{j} t$ with $\mu_{j}$ as the service rate. State of queuing system on start of $t$ duration is denoted by $Q_{i j}(n, t-1)$, and the state of queuing system on the end of $t$ duration is $Q_{i j}(n, t)$. Meanwhile, on the start of $t$ duration, remaining of packets in a queue is expressed as below.

$$
N_{t}=Q_{i j}(n, t-1)-C_{t}
$$

Meanwhile, free slot in a buffer queue is stated as follows.

$$
F_{t}=B-N_{t}=B-\left\{Q(n, t-1)-C_{t}\right\}
$$

Now, let us focus on the arrival of packet for $t$ of moment.

* If, $A_{t} \leq F_{t}$, the entire of packet arrival enter into the queue, so that packet population in a queue became $Q_{i j}$ $(n, t)=N_{t}+A_{t}$.

- If, $A_{t}>F_{t}$, packet entering to a queue is just as much as $F_{t}$, whereas rest of packet $\left(A_{t}-F_{t}\right)$ experience a loss.

* If, $N_{t}=B$, meaning that $F_{t}$ is zero (0), thereby the entire of packet arrival $\left(A_{t}\right)$ experience a loss.

When an arrival of packets is on case of $A_{t} \leq F_{t}$, then the arrival rate of packet entering to a queue buffer is $a_{m}^{j} \lambda_{j}$. However, while an arrival of packets on case of $A_{t}>F_{t}$ and $N_{t}=B$, the arrival rate of packet entering to a queue buffer is considered to be equal to zero (0). On the last both cases as mentioned above, packet loss does occur, so that packets reaching the receiver are incomplete and considered as invalid, thus retransmission is needed. Therefore, the arrival rate of packets entering queue buffer can be expressed as below.

$$
A=\left\{\begin{array}{l}
a_{m}^{j} \lambda_{j} \ldots \ldots ; A_{t} \leq F_{t} ; A<\mu_{j} ; N_{t}<B \\
0 \ldots \ldots \ldots . . ; A_{t}>F_{t} ; A \geq \mu_{j} ; N_{t}=B
\end{array}\right.
$$

As the previously mentioned, that state of packet queuing system is continuously dynamic. For more detailed review on the queuing system model, let us revisit the state of the queue system in " $j "$ phase, after transitions from " $i$ " phase, in a moment $(\delta)$. The queue system state is affected by initial condition probability and arrival probability $\left(a_{m}^{j} \lambda_{j} \delta\right)$, departure probability $\left(\mu_{j} \delta\right)$, and phase transition probability of the queue system, from " $i "$ to $" j "$ phase as $\left(r_{i j} \delta\right)$. By equation (3) and affecting factors as mentioned above, state of queuing system model can be expressed as follows.

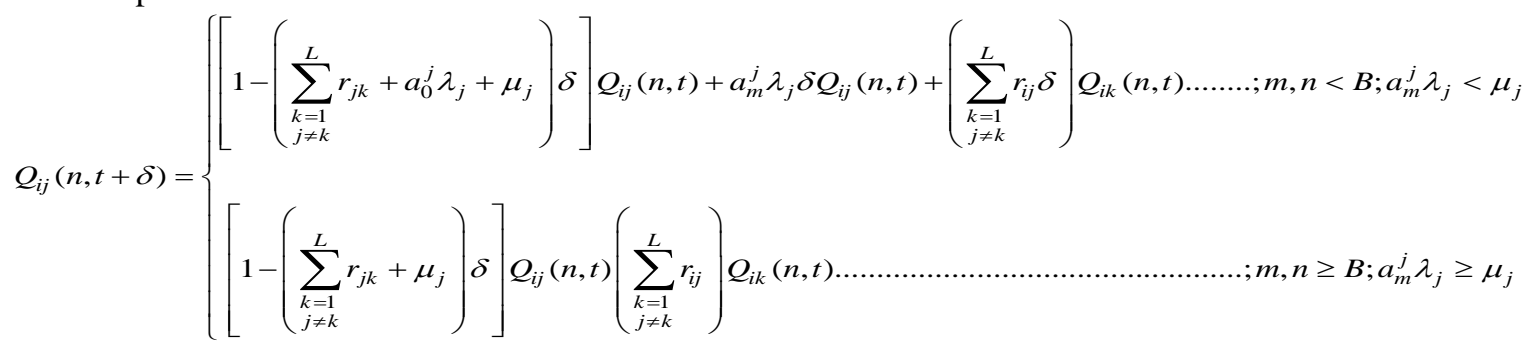

\subsection{Transition State of Queuing System}

State of queuing system is continuously varying at random from one state to the next. This condition can be described by a state transition in Markov chain diagram as shown in Figure 4. Further, state transition probability of queuing system is obtained from the difference of $Q_{i j}(n, t+\delta)$ to $Q_{i j}(n, t)$ on both sides of equation (4) and then divided by $\delta$ on the condition "limit $\delta \rightarrow 0$ ", thus equation (5) is obtained as follows: 


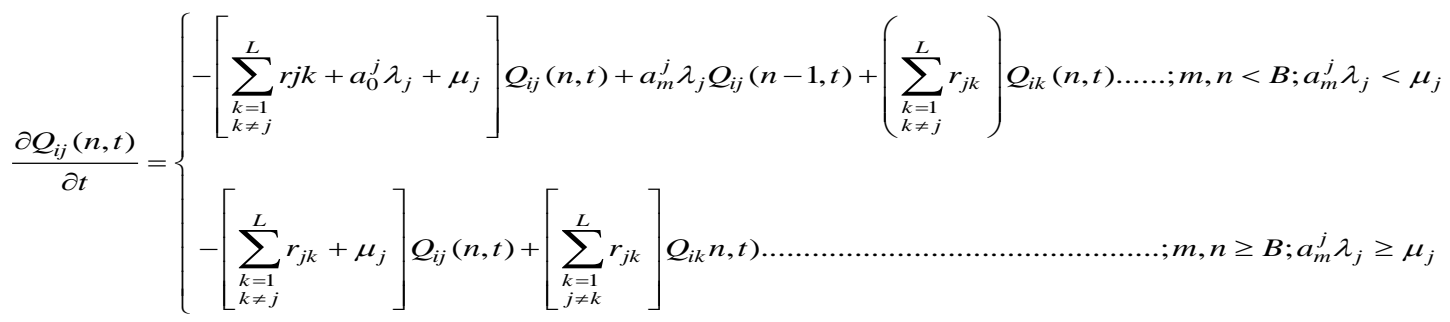

Briefly, the form of (5) could be denoted by (6) as follows.

$$
\frac{\partial Q_{i j}(n, t)}{\partial t}=Q_{i j}(n, t) \Gamma
$$

Referring to (6) " $i$ " and " $j "$, are phases of a queue system state, and $n=0,1,2, \ldots B$ shows a number of packets in the queue buffer, where $B$ is the maximum capacity of queue buffer size. Furthermore, $\Gamma$ is an infinitesimal generator matrix of state transition probability of the queue system with finite buffer. $\Gamma$ matrix is as shown as follows.

$$
\Gamma=\left[\begin{array}{cccccc}
D_{0} & D_{1} & D_{2} & D_{3} & \cdots & D_{B} \\
0 & D_{0} & D_{1} & D_{2} & \cdots & D_{(B-1)} \\
0 & 0 & D_{0} & D_{1} & \cdots & D_{(B-2)} \\
\vdots & \vdots & \vdots & \ddots & \ddots & \vdots \\
0 & 0 & 0 & \cdots & D_{0} & D_{1}
\end{array}\right]
$$

Related to above matrix, each row of the matrix could be described as follows:

* Row 1 , shows the queue state transition in a buffer generated by $D_{0}, D_{1}, \ldots D_{B}$ elements, from "empty-buffer as initial-state", after the departure of packet.

* Row 2, depicts the queue state transition in buffer generated by $D_{0}, D_{1}, \ldots, D_{(B-1)}$ elements, from "one (1) packet in buffer (indicated by zero (0) for the first column) as initial-state", after the departure of packet.

* Row 3, exposes the queue state transition in buffer generated by $D_{0}, D_{1}, \ldots, D_{(B-2)}$ elements, from "two packets in buffer (indicated by zero (0) for the first two columns) as initial-state", after the departure of packet.

* Row 4, up to one (1) row before the last row, shows a state transition pattern as well as on three (3) rows of matrix mentioned above, namely "the increase on total residual packets remaining in a queue buffer as initial-state", so that "decreases the amount of the arrival of packets that can enter a queue".

* Finally, the last row of matrix express the queue state transition in a buffer generated by $D_{0}, D_{l}$ elements, from "one (1) slot of empty-buffer, as initial-state", after the departure of packet. $D_{l}$ element at the last row and last column of the matrix shows there is just one (1) packet arrival that leads to full state of the buffer.

Further, the related matrix elements could be described as follow:

* $D_{0}$, shows elements of the transition matrix $\Gamma$ containing the drive of phase transition process of packet of queue system and departure of packets, but without the arrival of packets. $D_{0}$ is a matrix such as denote in (8).

* $\quad D_{m}$, with $(m=1,2, \ldots,(B-1), B)$ exposes the matrix element $\Gamma$ containing the drive of transitions as the packet population changes in the buffer queue by the batch arrival of packet by $m$ batch size. $D_{m}$ is a matrix shown by (9).

* In particular, an element of the last column of each matrix's row indicates the arrival of the group packet that results in full buffer queue, in a way that the arrival of next packets leads the system into overflow phase.

$D_{0}$ is expressed by a matrix as below.

$$
D_{0}=\left[\begin{array}{cccc}
-\sum_{j=2}^{L} r_{1 j}-a_{0}^{1} \lambda_{1}-\mu_{1} & r_{12} & \ldots & r_{1 L} \\
r_{21} & -\sum_{\substack{j=1 \\
j \neq 2}}^{L} r_{2 j}-a_{0}^{2} \lambda_{2}-\mu_{2} & \ldots & r_{2 L} \\
\vdots & \vdots & \ddots & \vdots \\
r_{2 L} & r_{L 2} & \ldots & -\sum_{j=1}^{L-1} r_{L j}-a_{0}^{L} \lambda_{L}-\mu_{L}
\end{array}\right]
$$


Whereas, $D_{m}$ is expressed in a matrix as follows.

$$
D_{m}=\left[\begin{array}{cccc}
a_{m}^{1} \lambda_{1} & 0 & \cdots & 0 \\
0 & a_{m}^{2} \lambda_{2} & \cdots & \cdots \\
\vdots & \vdots & \ddots & 0 \\
0 & 0 & 0 & a_{m}^{L} \lambda_{L}
\end{array}\right]
$$

Figure 3 shows the Markov chain diagram to illustrate the transition state of packet queuing system, which has been declared by the BMAP/M/1/B, and shown in mathematically by the equation (4), (5) and (7). Markov chain diagram shows the state transition of packets population on a queue buffer that occurred in three (3) phases of queue service state by slave station.

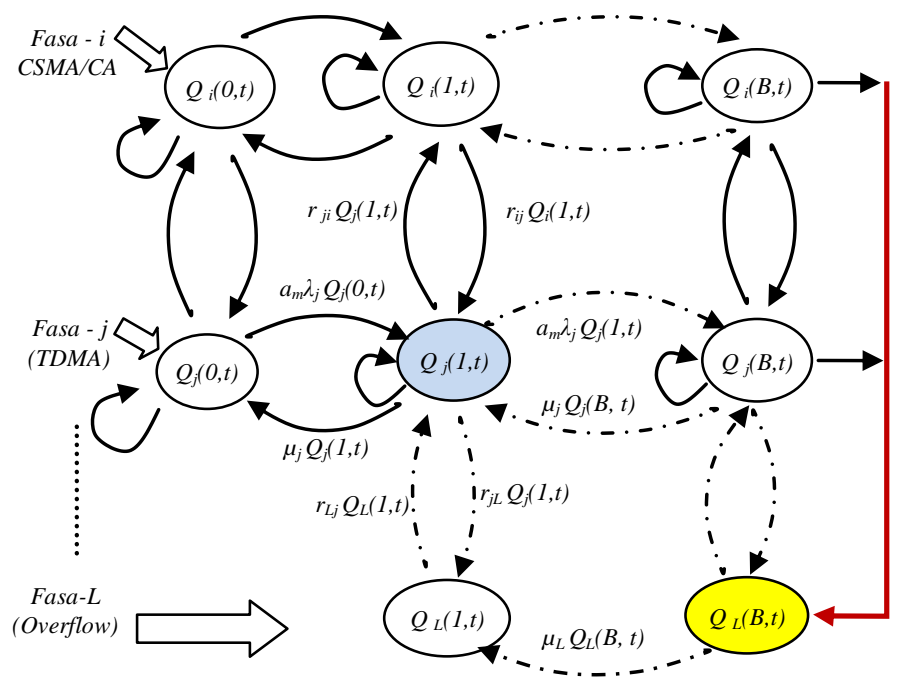

Fig. 3. State Transition Diagram of Batch Markov Arrival Process of Queue system on Limited Buffer Capacity

\section{Packet Queuing System Performance on Saturated Condition}

Considering a Markov chain diagram and suppose a queue system state on " $j$ " phase. If $n$ is packet population in a queue buffer, with $n=[0,1,2, \ldots, B]$, and while $n$ extremely near the $\mathrm{B}$ value, then a queue population will be close to full buffer. Consequently, queuing system reaches a saturated-state that leads to packet loss, thus lowering the queue system performance. Hence, our use of packet loss as an indicator of the queuing system performance is due to the notion that the increased number of packet loss can potentially decrease the integrity of data delivered through a Broadband PLC's slave station. As a result, we proceed by the analysis the queue of packet traffic on saturated conditions to obtain the packet loss. To retrieve the packet loss, furthermore, the key is discovering packet queue population in a steady-state, when the buffer condition is full.

\subsection{Analysis of Saturated Queue System}

Considering $a_{m}^{j} \lambda_{j}$ is the rate of packet arrival entering the queue buffer, while $\mu_{j}$ is the average of service rate over the output transmission channel of a queue system. It is defined that $\rho_{j}$ is a ratio of arrival rate to service rate, also called as network load. Corresponding to Poisson's process, probability of packet population in a queue buffer for $n^{\text {th }}$ packet arrival in the steady state is expressed as below.

$$
Q_{n}=Q_{0} \prod_{n=1}^{N} \frac{a_{m}^{j} \lambda_{(n-1), j}}{\mu_{n, j}}
$$

with $\frac{a_{m}^{j} \lambda_{(n-1), j}}{\mu_{n, j}}=\rho_{j}$, then equation (10) turns into (11) as follows.

$$
Q_{n}=Q_{0} \rho_{j}^{n}
$$


Where, $Q_{0}$ is an initial state of packet population probability in a queue buffer. $Q_{0}$ derived from normalization of the total sum of the $Q_{n}$ in equation (11) for $n$ to finite $N$, as expressed as follows.

$$
\sum_{n=0}^{N} Q_{n}=Q_{0}\left(1+\rho_{j}+\rho_{j}^{2}+\ldots+\rho_{j}^{N}\right)=1
$$

Equation (12) describes the packets population growth in a finite buffer of queuing system. Factor of limited geometric series in equation (12) can be expressed by the approach [9] as below.

$$
\left(1+\rho_{j}+\rho_{j}^{2}+\ldots+\rho_{j}^{N}\right) \approx \frac{1-\rho_{j}^{N+1}}{1-\rho_{j}}
$$

In line with the condition stated in (12) and the approach expressed in (12), thus $Q_{0}$ can be stated as follows.

$$
Q_{0}=\frac{1-\rho_{j}}{1-\rho_{j}^{N+1}}
$$

By substituting the $Q_{0}$ into (11), and for the value of $n=N=B$, then $Q_{n}$ becomes as follows.

$$
Q_{B}=Q_{0} \rho^{B}=\frac{(1-\rho) \rho^{B}}{\left(1-\rho^{B+1}\right)}
$$

Equation (15) shows the probability condition of packet population in a queue buffer, when a number of packet populations $(n)$ in a buffer equals to $B$, therefore, packet population at full condition.

\subsection{Packet Loss on Saturated Queue System of Packet Traffic}

When a queue buffer is full, next packet arrivals into a queue will lead the queue system to overflow phase. Accordingly, each packet arrival to a queue will encounter packet loss probability (PL) which is equal to equation (15). Thus, packet loss probability of queuing system can be obtained as follows.

$$
P_{L}=\frac{(1-\rho) \rho^{B}}{\left(1-\rho^{B+1}\right)}
$$

Form (16) shows dependence of the packet-loss probability on the arrival rate, departure rate, and queue buffer capacity.

\section{Numeric Result}

In this section, we show the calculation and numeric expression based on the analysis provided on section $\mathrm{V}$, to view saturation effects of queuing systems due to limited queue buffer capacity. Our perform a numerical experiment to observe the tendency of packet loss probability to packet arrival rate by taking into account both service rate and buffer capacity variations of a queue. Parameters of a queue system used to generate graphical expressions are in table 1 as follows.

Table 1. Parameters and Constants

\begin{tabular}{|c|c|c|}
\hline \multicolumn{2}{|c|}{ Parameters } \\
\hline $\begin{array}{c}\text { Rate of Arrival } \\
\text { (Mbps) }\end{array}$ & $\begin{array}{c}\text { Buffer }(B) \\
(\text { Mbit })\end{array}$ & $\begin{array}{c}\text { Sevice rate } \\
(\text { Mbps })\end{array}$ \\
\hline \multirow{3}{*}{14 s/d 150 } & 16 & 80, \\
\cline { 2 - 2 } & 32 & 100, \\
\cline { 2 - 2 } & 64 & 120, \\
\cline { 2 - 2 } & 128 & 135 and 150 \\
\hline
\end{tabular}

Figure 4 shows the probability of random packet loss upon 1,000 random arrivals of packet from 14 to 150 Mbps, with 64 Mbit buffer capacity, and at 100 Mbps departure rate.

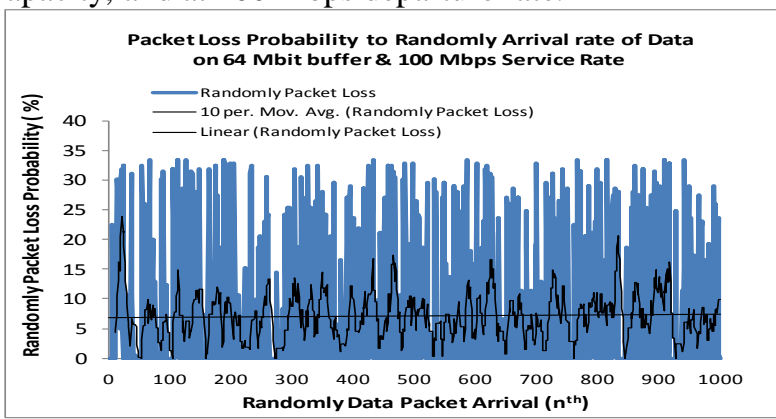

Fig. 4. Packet loss probability to randomly arrival rate of packet on 64 Mbit buffer, 100 Mbps Service rate 
Figure 5 exposes the packet loss probability to packet arrival rate in a queue with limited buffer capacity, and at 100 Mbps departure rate. Buffer capacity is observed at 16, 32, 64, and 128 Mbit to view packet loss probability over packet arrivals with varied buffer capacity and fixed service rate of a queue. Figure 6 depicts the packet loss probability to packet arrival rate at $64 \mathrm{Mbit}$ of buffer capacity in a queue. Varied service rate is provided to examine its effect on packet loss probability to packet arrival rate, with fixed buffer capacity.

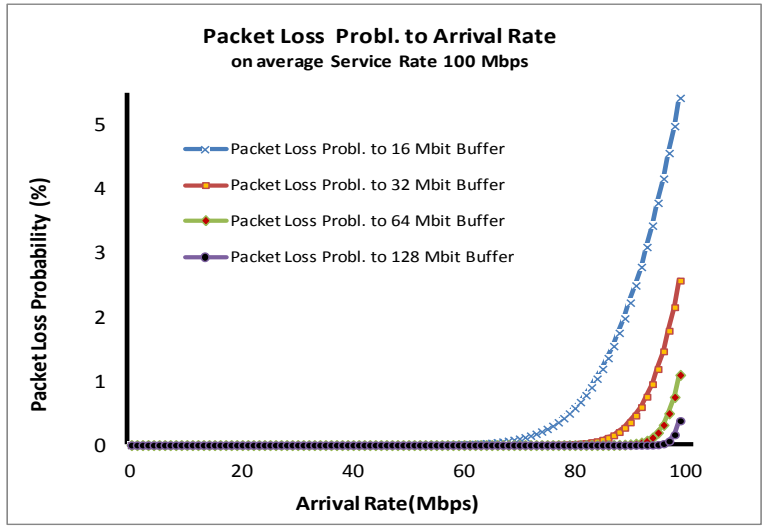

Fig. 5

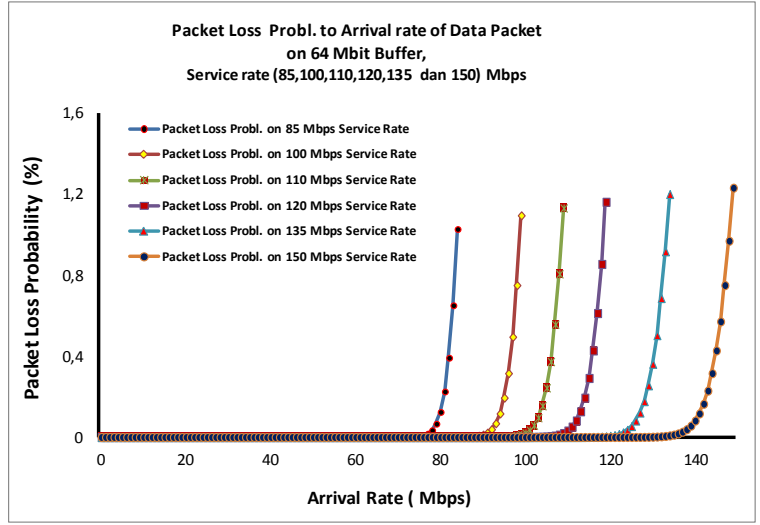

Fig. 6

Fig.5. Packet loss probability to data packet arrival rate on $100 \mathrm{Mbps}$ Service rate, and 4 kinds Buffer Capacity

Fig. 6. Packet loss Probability to data packet arrival rate on 64 buffer Capacity, and Service rate $50 \mathrm{Mbps}$

\subsection{Packet Loss Analysis on Saturated Condition}

Figure 4, shows a numerical experiments result of a packet queue on 64 Mbit buffer capacities and 100 Mbps service rate. For every 1,000 random arrivals of packets are varied between 14 - 150 Mbps, randomly packet loss probability occurs from zero (0) to $33.5 \%$, thus the average packet loss probability obtained is around $6.75 \%$. This meaning that average of data integrity reaches up to $93.25 \%$.

Figure 5 is a numerical experiment curve exposes the packet loss probability on the arrival rate of packets in queuing system with 100 Mbps service rate and for 16, 32, 64 and 128 Mbit varied buffer capacity. When the buffer capacity is at $16 \mathrm{Mbit}$, the packet loss probability is $5.42 \%$; and when buffer capacity is at 128 Mbit, it results in $0.38 \%$ packet loss probability. The queue system with lower buffer capacity to packet arrival rate, there is gets saturated more quickly than the queue system with higher buffer capacity.

Figure 6 depicts of numerical evaluation results of packet queuing systems. It shows the packet loss probability to packet arrival rate with $64 \mathrm{Mbit}$ buffer capacity, and service rate varies at $85,100,110,120,135$ and 150 Mbps. Packet loss probability of less than $0.1 \%$ is acquired when the packet arrival rate is $80 \mathrm{Mbps}$ or less, for all of service rate values. However, packet loss probability is fairly large when arrival rate is very close to service rate, even up to $100 \%$, when arrival rate exceeds all the service rate values.

\subsection{Parametric Analysis for Improvement of Queuing System Performance}

Increasing buffer capacity or service rate in a queue system may reduce the packet loss probability and improve the queue system performance. However, there are two options to consider as which of the two can give more significant reduction of packet loss probability. As a result, we need to observe the effect of either an increase of service rate or the addition of buffer capacity to the packet loss probability. Accordingly, we performed a numeric evaluation by two schemes resulting as follows.

1) Fixed buffer capacity at $64 \mathrm{Mbit}$ with an average arrival rate at $92 \mathrm{Mbps}$, while the service rate varies.

2) Fixed service rate at $100 \mathrm{Mbps}$ with an average arrival rate at $92 \mathrm{Mbps}$, while the buffer capacity varies.

Table 2. Parameters for Numeric calculation of packet loss to Varied of Service Rate and Buffer

\begin{tabular}{|c|c|r|r|r|r|r|}
\hline \multirow{2}{*}{$\begin{array}{c}\text { "Fixed" Buffer Capacity to } \\
\text { "Varied" Service Rate }\end{array}$} & Buffer capacity (Mbit) & \multicolumn{5}{|c|}{64} \\
\cline { 2 - 7 } & Service Rate (Mbps) & 100 & 105 & 110 & 115 & 120 \\
\hline \hline \multirow{2}{*}{$\begin{array}{c}\text { "Fixed" Buffer Capacity to } \\
\text { "Varied" Service Rate }\end{array}$} & Service Rate(Mbps) & \multicolumn{5}{|c|}{100} \\
\cline { 2 - 8 } & Buffer Capacity (Mbit) & 64 & 80 & 96 & 112 & 128 \\
\hline
\end{tabular}

Pertain to the analysis obtained on section 5.2, packet loss probability of a queue decreases when buffer capacity adds or service rate increases, and the trend is graphically as shown in Figure 7 and 8 below. 


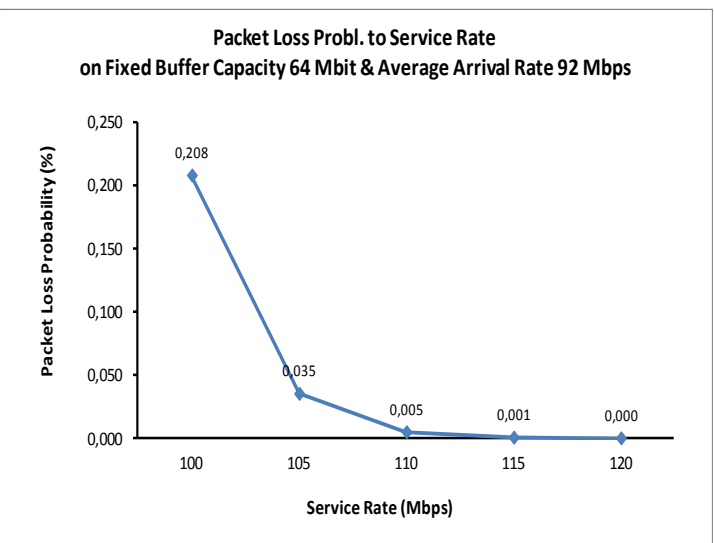

Fig. 7

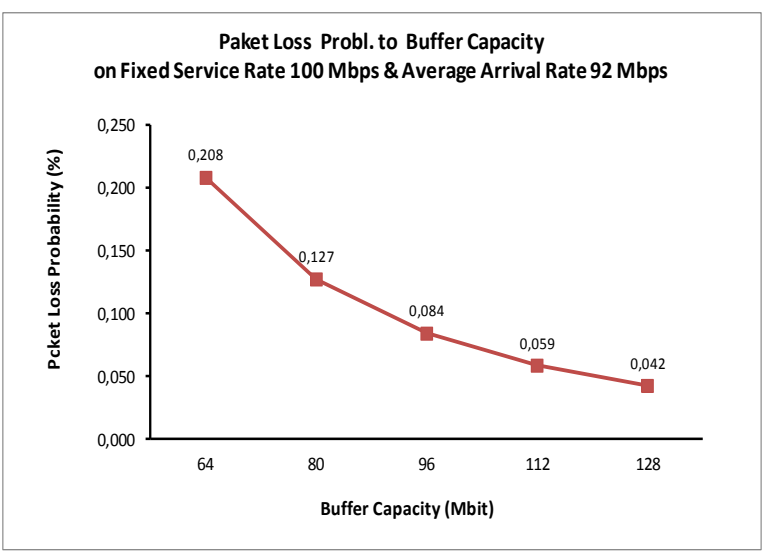

Fig. 8

Fig.7. Packet Loss Probability to Service rate on average arrival rate $92 \mathrm{Mbps}$

Fig. 8. Packet Loss Probability to Buffer capacity at an average of arrival rate $92 \mathrm{Mbps}$

With regard to Figures 7 and 8, the first of two sampling on both curves shows that packet loss probability decreases by -0.0346 slope against the increase of service rate of a queue. While at the addition of buffer capacity of a queuing, packet loss probability decreases by -0.0051 slope. Therefore, the increase of service rate is more significant than the addition of buffer capacity in reducing packet loss.

In other words, to improve queuing system performance, increasing service rate is more significantly effective than adding buffer capacity. In addition, adding capacity of internal buffer of a queue system requires by adding extra hardware. Adding buffer capacity in a large number of units could be expensive. Meanwhile, increasing queue service rate can be made through software technique, which even for huge quantity, the financial cost of increasing service rate is less than that of adding buffer capacity.

\section{Conclusion}

The packet traffic passing through the slave-station of Broadband PLC network with 100 Mbps service rate, 64 Mbit buffer capacities, and between $14 \mathrm{Mbps}$ to $150 \mathrm{Mbps}$ random arrival rate causes packet loss between zero (0) to $33.5 \%$, with the average loss of $6.75 \%$. Given the arrival rate is between $80 \mathrm{Mbps}$ to 90 Mbps, adding the buffer capacity from 16 Mbit to 32 Mbit, 64 Mbit and 128 Mbit on the queue system can reduce the packet loss probability approximately $0.1 \%$. However, if arrival rate exceeding service rate, addition of buffer capacity to a queue is worthless to reduce of packet loss probability.

The packet traffic passing through the slave-station of Broadband PLC network with 64 Mbit buffer capacities, arrival rate between $80 \mathrm{Mbps}$ to $90 \mathrm{Mbps}$, packet loss probability decreases more significantly than adding the buffer capacity from 16 Mbit to 32 Mbit, 64 Mbit and 128 Mbit. Such decrease occurs when the service rate is increased gradually for $85,100,110,120$, and 135 to $150 \mathrm{Mbps}$. On the packet traffic with 64 Mbps buffer capacity and average of arrival rate at $92 \mathrm{Mbps}$, the addition of service rate for $100 \mathrm{Mbps}$ to 105 Mbps has reduced the packet loss by 6.8 times lower than adding buffer capacity from 64 Mbit to 80 Mbit with fixed service rate $100 \mathrm{Mbps}$.

Further research work is to design control mechanisms to regulate the parameters of output transmission channel of slave-station to increase the service rate of a packet queue. The objective is to decrease packet loss and to enhance the packet queue performance. Adaptive control scheme can be embedded on to the control part of Medium Access Control (MAC) layer on slave station of Broadband PLC network.

\section{Acknowledgements}

We thank so much to, the Department of Electrical Engineering, University of Indonesia, the Directorate General of Higher Education of the Republic Indonesia, and the Telkom Education Foundation (YPT) - Telkom Institute of Technology, which has supported this research.

\section{References}

[1] Li.Fangxing, Qiao.W, Sun.H, Smart Transmission Grid : Vision and Framework, IEEE Transaction on Smart Grid, Vol .1, No.2, September 2010 .

[2] L Qingwen,Z.Shengli, B. Georgios, Queuing With Adaptiv Modulation and Coding Over Wireless Links; Cross-Layer Analysis and Design. IEEE Transaction on Wireless Communication. Vol.4.No.3.May 2005.

[3] B.S Aggelos, T.A. Georgios, L.P Dimitris, B.G. Anastasias, Selective Automation Upgrade in Distribution Networks Toward a Smarter Grid. IEEE Transaction on Smart Grid. Vol.1. No.3. December 2010.

[4] Rogers.K.M, K. Ray, K. Himanshu, A Angel, L Aquino, J Thomas, An Authenticated Control Framework for Distributed Support on the Smart Grid. IEEE. Transaction on Smart Grid Vol.1.No.1. June 2010 
[5] Yoon. S-G, Yun .J, Bahk S, Adaptive Contention Window Mechanism for Enhanching Throughput in HomePlug AV Network , 14244-1457-1/08/ IEEE. 2008.

[6] N-G James, W Robert : "Control and Automation of Electrical Power Distribution Systems" ; CRC Press Taylor \& Francis Group, 6000 Broken Sound Parkway NW, Suite 300. Boca Raton/London/New York. 2007.

[7] Hayes. J. F, Ghanes Babu.T.V.J," Modelling and Analysis of Telecommunications Networks:, John Wiley \& Sons. 2004

[8] H.Hrasnica, A. Haidine and R.Lehnert; "Broadband Powerline Communications_Network Design", John Wiley \& Sons, 2004.

[9] Kreyszig E : “Advanced Engineering Mathematics”, by John Willey \& Sons. Inc. 1988

[10] L.LiPing: "Performances and Quality of Service of PLC Network for MV and LV distribution systems", Thesis of Doctorat de I' Institut National Polytechnique de Lorraine. 2006.

[11] Galli.S, Scaglione. A, Wang.Zh , "For the Grid and Through the Grid : The Role of Power Line Communication in the Smart Grid", Vol.: 99 ; Issue:6; Proceeding of the IEEE, June 2011.

[12] Ser. Wah Oh : "Congitive Power Line Communication System for Multiple Channel Access"; Proc.on IEEE-ISPLC 2009, Dresden, Germany.29 March - 1 April 2009.

[13] P. Amirshahi and M. Kavehrad, “Transmission Channel Model and Capacity of Overhead Multi-conductor Medium-Voltage Power-lines for Broadband Communications," IEEE Consumer Communications \& Networking Conference, Las Vegas, Nevada, January 2005.

[14] T. Mastrangelo:" Ensuring ESA Capabilities during Network Transformation", An Aztek Networks ${ }^{\mathrm{TM}}$ White Paper ; The Windsor Oaks Group LLC 2007.

[15] HomePlug AV White Paper, HomePlug ${ }^{\circledR}$ Powerline Alliance, Inc. All Right Reserved. Copyright@2005. 